\title{
Efeito de diferentes soluções irrigadoras na microdureza superficial Knoop de dentes artificiais
}

\author{
Effect of different irrigation solutions on the Knoop surface microhardness of \\ artificial teeth
}

\author{
Paola Tentardini BAINYa (D), Simone Bonato LUISI $^{\mathrm{a}}{ }^{(1)}$, Francisco MONTAGNER $^{\mathrm{a}}$ (1), \\ Tiago André Fontoura de MELO ${ }^{\mathrm{a} *}$ [] \\ aUFRGS - Universidade Federal do Rio Grande do Sul, Faculdade de Odontologia, Porto Alegre, RS, Brasil
}

Como citar: Bainy PT, Luisi SB, Montagner F, Melo TAF. Efeito de diferentes soluções irrigadoras na microdureza superficial Knoop de dentes artificiais. Rev Odontol UNESP. 2019;48:e20190052. https://doi.org/10.1590/1807-2577.05219

\begin{abstract}
Resumo
Introdução: Dentes artificiais têm sido empregados para a prática de ensino pré-clínico. Porém, não se sabe o comportamento dos mesmos frente aos diferentes procedimentos realizados durante um tratamento endodôntico simulado. Objetivo: Avaliar a microdureza de réplicas de dentes humanos, frente a diferentes soluções irrigadoras. Material e método: Quarenta e cinco incisivos centrais superiores artificiais de três marcas comerciais nacionais (dentes " $A$ ", " $B$ " e " $C$ ") e 15 incisivos humanos foram utilizados. As amostras foram seccionadas a $5 \mathrm{~mm}$ do ápice dentário. Os fragmentos radiculares foram fixados em resina para posterior teste de microdureza. Os ensaios da microdureza Knoop foram realizados em dois momentos: momento 1 - microdureza inicial; momento 2 - microdureza final, que foi feita após o uso das soluções irrigadoras (Cloreto de Sódio a 0,9\%, Hipoclorito de Sódio a 2,5\% e Gluconato de Clorexidina a 2\%). As endentações deixadas na superfície da amostra foram mensuradas. O valor obtido para microdureza de cada amostra foi feita com base na média de três endentações. Para análise estatística, foram utilizados os testes ANOVA e Teste t pareado, com nível de significância de 5\%. Resultado: Os dentes artificiais " $\mathrm{A}$ " e "C" apresentaram valores inferiores de microdureza quando comparados ao dente humano $(\mathrm{P}=0,05)$. Não houve diferença estatística entre os dentes artificiais " $\mathrm{B}$ " e o dente humano ( $\mathrm{P}=0,2428)$. Quanto ao uso das diferentes soluções irrigadoras, também não houve diferença na microdureza das peças dentárias. Conclusão: Embora a solução irrigadora não tenha influenciado a microdureza, os dentes artificiais apresentaram valores de dureza inferiores aos do dente humano.
\end{abstract}

Descritores: Educação odontológica; treinamento endodôntico pré-clínico; dentes artificiais; microdureza.

\begin{abstract}
Introduction: Artificial teeth have been used for preclinical teaching practice. However, the behavior of the same is not known in relation to the different procedures performed during a simulated endodontic treatment. Objective: To evaluate the microhardness of replicates of human teeth, in front of different irrigating solutions. Material and method: Forty-five artificial upper central incisors of three national trade marks (teeth "A", "B" and "C") and fifteen human incisors were used. Samples were sectioned $5 \mathrm{~mm}$ from the apex. The root fragments were fixed in resin for later microhardness test. The Knoop microhardness tests were performed in two moments: moment 1 - initial microhardness; moment 2 - final microhardness, which was done after the use of the irrigating solutions $(0.9 \%$ sodium chloride, $2.5 \%$ sodium hypochlorite and $2 \%$ chlorhexidine gluconate). The indentations left on the sample surface were measured. The obtained value for microhardness of each sample was made based on the average of three indentations. ANOVA and Paired t-test were used for statistical analysis. Significance level of $5 \%$. Result: Artificial teeth " $A$ " and "C" presented lower values of microhardness when compared to human teeth $(P=0.05)$. There was no statistical difference between the artificial teeth " $\mathrm{B}$ " for the human $(\mathrm{P}=0.2428)$. Regarding the use of different irrigation solutions, there was also no difference in the microhardness of dental pieces. Conclusion: Although the irrigating solution did not influence the microhardness, the artificial teeth presented values of hardness inferior to the human tooth.
\end{abstract}

Descriptors: Dental education; preclinical endodontic training; artificial teeth; microhardness. 


\section{INTRODUÇÃO}

A realização das atividades laboratoriais nas disciplinas pré-clínicas em Odontologia tem como objetivo proporcionar aos alunos um treinamento prático manual, fundamentado nos conhecimentos teóricos apresentados e discutidos em sala de aula previamente ao início das atividades clínicas.

O emprego de dentes humanos em tais atividades, diante da atual legislação brasileira, está relacionado à utilização de órgãos e tecidos. Surgem então questões éticas relacionadas ao comércio ilegal, questões estruturais associadas à criação de biobancos e à forma de armazenamento desses dentes extraídos, além do controle de biossegurança no seu manuseio ${ }^{1,2}$.

Recentemente, foram introduzidas no mercado réplicas artificiais em material resinoso ou acrílico, que se apresentam como alternativa adequada para substituição dos dentes humanos em atividades de treinamento pré-clínico em Endodontia. Assim, se busca uma padronização nas características anatômicas, tais como comprimento, diâmetro e grau de curvatura, o que torna essas réplicas instrumentos valiosos tanto para o treinamento quanto para avaliação dos alunos. No entanto, surgem dúvidas quanto à sua capacidade em reproduzir as estruturas dentais humanas.

Em Endodontia, além das condições anatômicas, a dureza é outro fator importante que deve ser observado nos dentes artificiais. A obtenção de uma resistência ao corte e ao desgaste do dente artificial com uso dos instrumentos endodônticos representa um fato de extrema importância para que o aluno adquira a sensação tátil associada aos procedimentos, tornando-a mais fidedigna a uma situação clínica real. Outro fator que também deve ser analisado quanto ao quesito dureza dos materiais é a possível influência das soluções irrigadoras utilizadas na Endodontia sobre essa propriedade. Alterações na microdureza dos materiais poderiam estar associadas à interação entre os mesmos - pois, geralmente, são formados por compostos orgânicos - e soluções com capacidade de dissolução de matéria orgânica. Até o presente momento, não há nenhum estudo prévio a este respeito com relação aos dentes artificiais. Em 2007, Oliveira et al. ${ }^{3}$ avaliaram o efeito da solução salina, do Hipoclorito de Sódio a $1 \%$ e do Gluconato de Clorexidina a $2 \%$ sobre a microdureza da dentina radicular humana após 15 minutos de irrigação. Apenas a Clorexidina e o Hipoclorito reduziram significativamente a microdureza da dentina do canal radicular a $500 \mu \mathrm{m}$ e $1.000 \mu \mathrm{m}$ da interface polpa-dentina, respectivamente.

O presente estudo tem como objetivo avaliar a microdureza de diferentes réplicas de dentes humanos para uso em atividades endodônticas laboratoriais de ensino, frente a diferentes soluções irrigadoras.

\section{MATERIAL E MÉTODO}

O presente estudo foi aprovado pelo Comitê de Pesquisa da Faculdade de Odontologia da UFRGS e pelo Comitê de Ética em Pesquisa da mesma instituição (Protocolo CAAE 89501218.0.0000.5347).

\section{Tipos de Dentes Testados}

A amostra foi constituída por sessenta incisivos centrais superiores, sendo 45 dentes artificiais para Endodontia e 15 dentes humanos extraídos.

Os dentes endodônticos artificiais foram adquiridos em três empresas comerciais nacionais. Os incisivos centrais superiores humanos foram obtidos a partir de pacientes maiores de 18 anos, de ambos os sexos, atendidos nas disciplinas de Cirurgia e Traumatologia 
Bucomaxilofaciais I e II Diurno e Noturno da UFRGS, que aceitaram efetuar doação dos mesmos. Os dentes humanos foram extraídos por motivos diversos, que não associados a essa pesquisa. Os dentes foram previamente radiografados para identificação dos seguintes fatores de exclusão: manipulação endodôntica prévia, presença de alterações anatômicas ao longo do canal radicular e fraturas radiculares. Após a realização dos procedimentos de limpeza com curetas periodontais, os dentes passaram por um processo de desinfecção, sendo imersos em um pote plástico contendo solução de Cloramina a 0,5\% (Seachem Laboratories, Madison, GA, USA) por 48 horas.

\section{Soluções Irrigadoras Testadas}

As soluções irrigadoras utilizadas foram o Cloreto de Sódio a 0,9\% (Eurofarma Laboratórios S.A., São Paulo, São Paulo, Brasil), Hipoclorito de Sódio a 2,5\% (Iodontosul - Industrial Odontológica do Sul LTDA., Porto Alegre, Rio Grande do Sul, Brasil) e o Gluconato de Clorexidina a 2\% (Maquira Indústria de Produtos Odontológicos S.A., Maringá, Paraná, Brasil).

\section{Divisão dos Grupos Experimentais}

Dentro de cada um dos quatro tipos de dentes testados, as amostras foram divididas aleatoriamente em três subgrupos experimentais (Quadro 1), usando-se a técnica de amostra casual simples por meio do programa Excel (Microsoft Excel, Microsoft, USA).

Quadro 1. Quadro demonstrativo dos grupos experimentais

\begin{tabular}{cccc}
\hline Grupo & n & Tipo de Dente & Solução Irrigadora \\
\hline G1 & 5 & Dente Humano & Cloreto de Sódio a 0,9\% \\
G2 & 5 & Dente Humano & Hipoclorito de Sódio a 2,5\% \\
G3 & 5 & Dente Humano & Gluconato de Clorexidina a 2\% \\
G4 & 5 & Dente Artificial "A" & Cloreto de Sódio a 0,9\% \\
G5 & 5 & Dente Artificial "A" & Hipoclorito de Sódio a 2,5\% \\
G6 & 5 & Dente Artificial "A" & Gluconato de Clorexidina a 2\% \\
G7 & 5 & Dente Artificial "B" & Cloreto de Sódio a 0,9\% \\
G8 & 5 & Dente Artificial "B" & Hipoclorito de Sódio a 2,5\% \\
G9 & 5 & Dente Artificial "B" & Gluconato de Clorexidina a 2\% \\
G10 & 5 & Dente Artificial “C" & Cloreto de Sódio a 0,9\% \\
G11 & 5 & Dente Artificial “C" & Hipoclorito de Sódio a 2,5\% \\
G12 & 5 & Dente Artificial "C" & Gluconato de Clorexidina a 2\% \\
\hline
\end{tabular}

\section{Preparo das Amostras Dentárias}

Inicialmente, as amostras dentárias foram cortadas a $5 \mathrm{~mm}$ do ápice dentário com uso de disco de aço diamantado dupla-face (KG Sorensen, Barueri, São Paulo, Brasil), perpendicular ao longo eixo do dente.

Os segmentos radiculares obtidos foram incluídos em blocos de resina acrílica autopolimerizável (JET, Clássico, Campo Limpo Paulista, São Paulo, Brasil), utilizando-se cilindros de PVC (Tigre S.A., Joinville, Santa Catarina, Brasil) com as dimensões de $2 \mathrm{~cm}$ de altura por $5 \mathrm{~cm}$ de diâmetro, de modo que a face cervical da raiz ficasse livre e nivelada à superfície da resina acrílica. 
Quando do início da reação exotérmica de polimerização da resina, os blocos foram imersos em água destilada à temperatura ambiente, a fim de evitar possíveis danos aos espécimes por ação do calor liberado.

A superfície da face cervical livre da raiz foi polida em uma politriz circular (DP-10, StruersPanambra, São Paulo, São Paulo, Brasil) com lixas de carbeto de silício em uma sequência da granulação mais grossa para a mais fina (Norton Abrasivos Brasil, São Paulo, São Paulo, Brasil). 0 polimento foi realizado utilizando-se a lixa de granulação 600 por 40 segundos, seguida pelas lixas 1.000 e 2.000 por 60 segundos cada. Em seguida, as amostras foram lavadas em água corrente e colocadas em um aparelho de ultrassom com água destilada, por 10 minutos, para remoção de resíduos superficiais.

\section{Ensaio de Microdureza Knoop}

A fim de permitir comparações entre as medidas de microdureza dos mesmos espécimes em diferentes momentos, houve a necessidade de se criar uma padronização de região dentro dos respectivos blocos de resina. A aferição foi movida em $200 \mu \mathrm{m}$ a cada momento de análise. Além disso, a posição estabelecida para aferição foi analisada previamente com uma lupa para se ter certeza de que a posição já não tivesse sido aferida anteriormente. Assim, cada bloco teve um de seus ângulos demarcados para referência de posição e, aos espécimes, foi atribuída uma ordem topográfica a ser seguida nas leituras.

Previamente à execução do teste de microdureza, cada metade dentária foi seca previamente com jato de ar, isento de óleo.

Os blocos foram levados ao microdurômetro (Microhardness Tester FM 700, Future Tech Corp., Tokyo, Japan), sendo obtidos valores de microdureza na unidade Knoop Hardness Number (KHN).

Três endentações foram efetuadas para cada espécime a $500 \mu \mathrm{m}$ da luz do canal radicular, utilizando-se carga perpendicular ao corpo de prova de $10 \mathrm{~g}$ por cinco segundos. Cada endentação teve as diagonais aferidas. Foi considerada a média dos valores de microdureza das três endentações para cada espécime.

As medidas da microdureza inicial (Md1) foram realizadas antes do uso dos irrigantes. Já a microdureza final (Md2) foi feita após o uso das soluções irrigadoras. Mechas de algodão embebidas com as substâncias testadas foram colocadas sobre a face cervical da raiz e no interior da luz de cada segmento radicular, permanecendo em contato com o material por dois minutos.

\section{Análise Estatística}

Os dados foram tabulados e submetidos à análise estatística no programa GraphPad Prism ${ }^{\circledR}$ (GraphPad Software, Inc., San Diego, CA, USA). 0 teste de Kolmogorov-Smirnov foi utilizado para avaliar a normalidade dos dados. Para comparação dos grupos quanto à microdureza, foi utilizada a Análise de Variância (ANOVA), seguida do teste de comparações múltiplas de Tukey, quando necessário.

Já para a comparação das diferentes soluções irrigadoras em relação aos tipos de dentes, foram utilizados o Teste t pareado, seguido do Teste pareado de Mann-Whitney, e ANOVA, seguido do Teste de Kruskal-Wallis. 0 nível de significância foi de 5\%.

A hipótese nula do estudo foi que não há diferenças estatísticas tanto em relação à microdureza dos diferentes dentes testados quanto à influência do tipo de solução irrigadora utilizada. 


\section{RESULTADO}

A média e o desvio padrão da microdureza superficial dos dentes testados no experimento estão expressos no Figura 1.

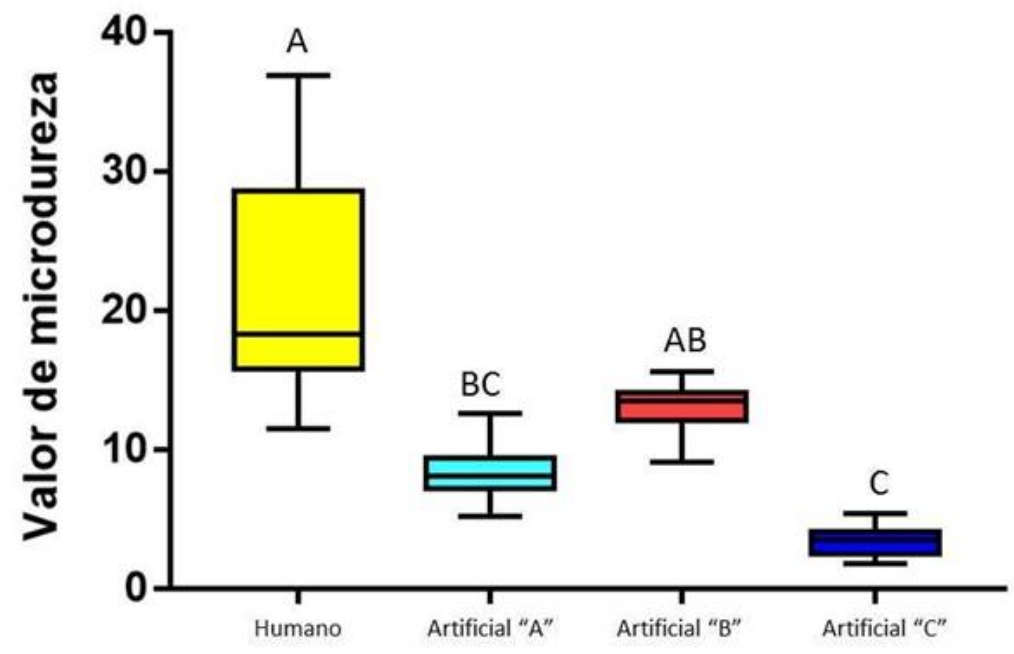

\section{Dentes testados}

Figura 1. Comparação das médias de microdureza superficial dos dentes testados. Médias seguidas de letras maiúsculas distintas na coluna diferem significativamente por meio da Análise de Variância, seguida do Teste de Tukey, no nível de significância de $5 \%$.

Na Tabela 1, estão expressos os resultados referentes à influência ou não do tipo de irrigante utilizado na microdureza superficial das diferentes peças dentárias.

Tabela 1. Microdureza superficial dos diferentes dentes testados antes e após o uso das soluções irrigadoras

\begin{tabular}{|c|c|c|c|c|c|c|c|c|}
\hline \multirow{2}{*}{$\begin{array}{l}\text { Soluções } \\
\text { Irrigadoras }\end{array}$} & \multicolumn{2}{|c|}{$\begin{array}{l}\text { Dente Humano } \\
\text { (Média } \pm \text { DP) }\end{array}$} & \multicolumn{2}{|c|}{$\begin{array}{l}\text { Artificial "A" } \\
\text { (Média } \pm \text { DP) }\end{array}$} & \multicolumn{2}{|c|}{$\begin{array}{l}\text { Artificial "B" } \\
\text { (Média } \pm \text { DP) }\end{array}$} & \multicolumn{2}{|c|}{$\begin{array}{l}\text { Artificial "C" } \\
\text { (Média } \pm \text { DP) }\end{array}$} \\
\hline & Antes & Depois & Antes & Depois & Antes & Depois & Antes & Depois \\
\hline Cloreto de Sódio 0,9\% & $\begin{array}{c}19,84 \text { Aa } \\
\pm 5,69\end{array}$ & $\begin{array}{l}16,65 \text { Аа } \\
\pm 4,90\end{array}$ & $\begin{array}{c}7,26 \text { Аа } \pm \\
1,33\end{array}$ & $\begin{array}{c}6,39 \text { Аа } \pm \\
2,16\end{array}$ & $\begin{array}{l}12,73 \text { Аа } \\
\pm 2,41\end{array}$ & $\begin{array}{l}13,12 \text { Аа } \\
\pm 1,74\end{array}$ & $\begin{array}{c}2,86 \text { Аа } \pm \\
1,43\end{array}$ & $\begin{array}{c}3,64 \mathrm{Aa} \pm \\
1,04\end{array}$ \\
\hline $\begin{array}{l}\text { Hipoclorito de Sódio } \\
2,5 \%\end{array}$ & $\begin{array}{c}21,95 \text { Aa } \\
\pm 8,27\end{array}$ & $\begin{array}{l}25,54 \text { Aa } \\
\pm 6,09\end{array}$ & $\begin{array}{c}9,32 \text { Аа } \pm \\
2,01\end{array}$ & $\begin{array}{c}8,61 \text { Aa } \pm \\
2,56\end{array}$ & $\begin{array}{l}12,68 \text { Аa } \\
\pm 2,04\end{array}$ & $\begin{array}{l}11,88 \text { Аа } \\
\pm 2,21\end{array}$ & $\begin{array}{c}3,12 \text { Аa } \pm \\
0,63\end{array}$ & $\begin{array}{c}3,85^{\mathrm{Aa}} \pm \\
1,08\end{array}$ \\
\hline $\begin{array}{l}\text { Gluconato de Clorexidina } \\
\qquad 2 \%\end{array}$ & $\begin{array}{c}23,2 \text { Аа } \pm \\
9,66\end{array}$ & $\begin{array}{l}25,42 \text { Аa } \\
\pm 8,32\end{array}$ & $\begin{array}{c}8,65 \text { Аa } \pm \\
1,87\end{array}$ & $\begin{array}{c}9,50 \text { Аa } \pm \\
2,02\end{array}$ & $\begin{array}{l}13,74 \text { Aa } \\
\pm 0,89\end{array}$ & $\begin{array}{l}13,35 \text { Аа } \\
\pm 1,27\end{array}$ & $\begin{array}{c}4,23 \text { Аа } \pm \\
0,77\end{array}$ & $\begin{array}{c}4,69 \text { Aa } \pm \\
0,56\end{array}$ \\
\hline
\end{tabular}

Médias seguidas de letras maiúsculas distintas na coluna diferem significativamente por meio do Teste One-way ANOVA, seguido do Teste de Kruskal-Wallis. Médias seguidas de letras minúsculas distintas na linha, dentro de cada um dos tipos de dentes analisados, diferem significativamente por meio do Teste t pareado, seguido do Teste pareado de Mann-Whitney. Nível de significância das análises de 5\%.

\section{DISCUSSÃO}

A realização de um correto treinamento pré-clínico busca garantir que os alunos realizem tratamentos endodônticos de boa qualidade, previamente ao atendimento de pacientes. Para isso, os dentes artificiais devem reproduzir as complexidades anatômicas e ter similaridades de dureza e radiopacidade dos dentes naturais. 
A hipótese nula de que não haveria diferença estatística na microdureza dos diferentes dentes testados foi rejeitada. No estudo, pode-se observar que os dentes artificiais apresentaram um menor valor de microdureza quando comparados a esse valor do dente humano, embora não tenha ocorrido diferença nos dados entre o incisivo artificial "B" e o dente humano $(P=0,2428)$. Os resultados demonstram uma grande variação na microdureza superficial em dentes humanos, o que pode ser devido a fatores, como idade dos participantes e alterações clínicas presentes antes do momento da coleta, tal como mineralização. De certa forma, a microdureza dos dentes humanos ainda não consegue ser reproduzida com precisão pelos dentes artificiais disponíveis, conforme constatado nos estudos de Nassri et al. ${ }^{4}$ e Luz et al. ${ }^{5}$.

Segundo Robberecht et al. ${ }^{6}$, a dureza insuficiente das réplicas dentárias irá resultar em sensações tácteis não realistas durante a realização do preparo químico-mecânico do canal radicular, em comparação aos dentes naturais humanos.

Nos estudos de Luz et al. ${ }^{5}$ e Bitter et al. ${ }^{7}$, pode-se observar que os alunos realizaram o tratamento endodôntico nos dentes artificiais num menor tempo do que em relação ao treinamento em dentes humanos. Essa constatação pode ser justificada não somente pela menor dureza do material como também pelas condições anatômicas dentárias, que não apresentam as dificuldades inerentes existentes nos dentes humanos, tais como atresias e calcificações dentinárias. Esse fato poderia favorecer um melhor desempenho, mas não reflete a realidade anatômica a ser observada em dentes artificiais.

Independentemente das soluções irrigadoras testadas (Cloreto de Sódio a 0,9\%, Hipoclorito de Sódio a 2,5\% e o Gluconato de Clorexidina a 2\%), não houve alteração no valor de microdureza apresentado pelos dentes. Não há na literatura nenhum estudo a este respeito com dentes artificiais. Porém, considerando-se os resultados observados em dentes humanos, há concordância com os achados de Ari et al. ${ }^{8}$ e Patil, Uppin'.

Por outro lado, Oliveira et al. ${ }^{3}$ observaram resultados diferentes do nosso estudo. Os autores verificaram que tanto o Hipoclorito de Sódio a $1 \%$ quanto o Digluconato de Clorexidina a $2 \%$ reduziram significativamente a microdureza da dentina do canal radicular, porém com um período maior que 15 minutos de exposição às soluções.

Segundo o estudo de Slutzky-Goldberg et al. ${ }^{10}$, quanto maior a concentração da solução irrigadora maior será a alteração causada sobre a microdureza dentinária. Os autores indicam que a diminuição da microdureza foi maior após irrigação com Hipoclorito a $6 \%$ do que a 2,5\%.

Acredita-se que a busca por outro material mais rígido e com valores de microdureza superiores aos dentes testados seja fundamental para se aproximar às condições existentes nos dentes humanos, o que irá proporcionar assim uma prática laboratorial mais fidedigna possível.

\section{CONCLUSÃO}

Embora não tenha ocorrido diferença estatística entre os dentes artificial "B" e o dente humano, pode-se concluir que os valores de microdureza dos dentes artificiais foram inferiores aos do dente humano.

Não houve diferença nos valores de microdureza com relação às diferentes soluções irrigadoras testadas.

\section{REFERÊNCIAS}

1. Carvalho C. Dentes na mira da ética. Rev Bras Odontol. 2001 Mar-Abr;58(2):108-11. 
2. Costa SM, Mameluque S, Brandão EL, Melo AEMA, Pires CPAB, Rezende EJC, et al. Dentes humanos no ensino odontológico: procedência, utilização, descontaminação e armazenamento pelos acadêmicos da UNIMONTES. Rev ABENO. 2007 Jan-Abr;7(1):6-12.

3. Oliveira LD, Carvalho CA, Nunes W, Valera MC, Camargo CH, Jorge AO. Effects of chlorhexidine and sodium hypochlorite on the microhardness of root canal dentin. Oral Surg Oral Med Oral Pathol Oral Radiol Endod. 2007 Oct;104(4):e125-8. http://dx.doi.org/10.1016/j.tripleo.2007.04.019. PMid:17656125.

4. Nassri MRG, Carlik J, Silva CRN, Okagawa RE, Lin S. Critical analysis of artificial teeth for endodontic teaching. J Appl Oral Sci. 2008 Jan-Feb;16(1):43-9. http://dx.doi.org/10.1590/S167877572008000100009 . PMid:19089288.

5. Luz DS, Ourique FS, Scarparo RK, Vier-Pelisser FV, Morgental RD, Waltrick SB, et al. Preparation time and perceptions of Brazilian specialists and dental students regarding simulated root canals for endodontic teaching: a preliminar study. J Dent Educ. 2015 Jan;79(1):56-63. PMid:25576553.

6. Robberecht L, Hornez JC, Dehurtevent M, Dufour T, Labreuche J, Deveaux E, et al. Optimization and preclinical perception of an artificial simulator for endodontic training: a preliminary study. J Dent Educ. 2017 Mar;81(3):326-32. PMid:28250039.

7. Bitter K, Gruner D, Wolf $\mathrm{O}$, Schwendicke F. Artificial versus natural teeth for preclinical endodontic training: a randomized controlled trial. J Endod. 2016 Aug;42(8):1212-7. http://dx.doi.org/10.1016/j.joen.2016.05.020. PMid:27469437.

8. Ari H, Erdemir A, Belli S. Evaluation of the effect of endodontic irrigation solutions on the microhardness and the roughness of root canal dentin. J Endod. 2004 Nov;30(11):792-5. http://dx.doi.org/10.1097/01.DON.0000128747.89857.59. PMid:15505513.

9. Patil CR, Uppin V. Effect of endodontic irrigating solutions on the microhardness and roughness of root canal dentin: an in vitro study. Indian J Dent Res. 2011 Jan-Feb;22(1):22-7. http://dx.doi.org/10.4103/0970-9290.79969. PMid:21525672.

10. Slutzky-Goldberg I, Maree M, Liberman R, Heling I. Effect of sodium hypochlorite on dentin microhardness. J Endod. 2004 Dec;30(12):880-2. http://dx.doi.org/10.1097/01.DON.0000128748.05148.1E. PMid:15564869.

\section{CONFLITOS DE INTERESSE}

Os autores declaram não haver conflitos de interesse.

\section{*AUTOR PARA CORRESPONDÊNCIA}

Tiago André Fontoura de Melo, UFRGS - Universidade Federal do Rio Grande do Sul, Faculdade de Odontologia, Departamento de Odontologia Conservadora, Rua Ramiro Barcelos, 2492, Bairro Santana, 90035-003 Porto Alegre - RS, Brasil, e-mail: tiago.melo@ufrgs.br

Recebido: Maio 17, 2019

Aprovado: Agosto 28, 2019 